\section{Case Reports in Oncology}

\title{
Aneurysmal Fibrous Histiocytoma: A Large Soft Tissue Tumor with Metastases Treated with Palliative Radiation Therapy and Targeted Therapy
}

\author{
Michael Chamberlin ${ }^{a}$ Sophia Khan ${ }^{b}$ Cristal Hernandez ${ }^{b}$ \\ Markku Miettinen $^{c}$ Jun S. Weid Javed Khan ${ }^{d}$ John Glod ${ }^{b}$ \\ Lindsay Rowe Aradhana Kaushal $^{f}$ \\ aRadiation Oncology Branch, Center for Cancer Research, National Cancer Institute, \\ Bethesda, MD, USA; ${ }^{b}$ Pediatric Oncology Branch, Center for Cancer Research, National \\ Cancer Institute, Bethesda, MD, USA; ' Laboratory of Pathology, Center for Cancer \\ Research, National Cancer Institute, Bethesda, MD, USA; dGenetics Branch, Center \\ for Cancer Research, National Cancer Institute, Bethesda, MD, USA; ${ }^{\text {eDepartment of }}$ \\ Radiation Oncology, Cross Cancer Institute, University of Alberta, Edmonton, AB, Canada; \\ fDepartment of Radiation Medicine, University of Kentucky, Lexington, KY, USA
}

\section{Keywords}

Aneurysmal fibrous histiocytoma - Radiation · Pazopanib · Protein kinase C inhibitor .

Midostaurin

\begin{abstract}
Aneurysmal fibrous histiocytoma (AFH) is a rare variant of cutaneous fibrous histiocytoma, with low malignant potential and infrequent metastatic progression. We present the case of a 19-year-old female with a large AFH of the neck metastatic to soft tissue and treated with radiation therapy and molecularly targeted therapy. To our knowledge, this is the first report describing either radiation therapy and palliation or the use of targeted therapy in this uncommon malignancy and can provide insight into future therapeutic strategies.
\end{abstract}




\section{Introduction}

Aneurysmal fibrous histiocytoma (AFH; also referred to as aneurysmal benign fibrous histiocytoma) is a rare variant of cutaneous cellular fibrous histiocytoma. It accounts for $0.3 \%$ of soft tissue sarcomas/borderline tumors, and is classified by the WHO under tumors of uncertain differentiation [1,2]. Excision alone is usually curative, and progression of cutaneous fibrous histiocytoma to metastatic disease is rare; however, the frequency is difficult to determine due to its potential overlap with deep fibrous histiocytoma, which has a $5 \%$ risk of metastasis [3,4]. The rarity of this tumor coupled with its uncommon malignant progression significantly limits the available data regarding treatment algorithms, especially in the metastatic setting.

We present the case of a young female with metastatic $\mathrm{AFH}$ who was treated with radiation therapy with a durable palliative response.

\section{Case Presentation}

A 19-year-old female from Gambia presented to our institution with a large, fungating soft tissue tumor seeking an opinion regarding diagnosis and treatment options. The patient first noted an asymptomatic superficial mass in the posterior occipital region of her right neck 10 years previously. For 2 years,this was treated with local indigenous methods. However, after continued progression she presented to a medical facility, and biopsy of the lesion initially was diagnosed as mixed cellularity Hodgkin's lymphoma. Her specific treatment history at that time was unavailable; however, the patient stated that she underwent chemotherapy but did not respond to treatment.

Six years after her initial presentation, she developed a second lesion on the superficial right anterolateral cheek, and a biopsy of this lesion was diagnosed as dermatofibrosarcoma. From the time of developing the second lesion, she underwent 6 surgical excisions of the lesions over a period of 3 years, all of which were complicated by significant bleeding. The lesions continued to recur superficially, without evidence of local invasion or metastases. She required multiple hospitalizations for tumor site infection and blood transfusions as a result of spontaneous superficial tumor bleeding.

At the time of presentation to our institution, she had developed symptoms related to tumor site infection and hemorrhage. She presented with swelling, pain, chronic bleeding, and odorous purulence (Fig. 1a). Significant pain led to poor oral intake and nutritional decline, and the patient also experienced trismus with an inter-incisal distance of $1 \mathrm{~cm}$ due to pain and involvement of the muscles of mastication. Staging demonstrated innumerable pulmonary nodules as well as numerous soft tissue nodules throughout the trunk (Fig. 1b, c). A pathology review of a surgical specimen from her previous surgery showed AFH (Fig. 3). An excisional biopsy was performed on a right hip nodule. The histologic appearance was identical to her primary lesion and showed features of aneurysmal fibrous histiocytoma. The tumor was negative for CD34, desmin, keratins, S-100 protein, and SMA. RNA sequencing followed by confirmatory RT-PCR and Sanger sequencing was performed and identified a LAMTOR1-PRKCD gene fusion previously identified in this tumor type. While the pulmonary lesions were felt to be most consistent with metastatic disease, the patient was also diagnosed with latent tuberculosis, complicating the diagnosis. Her treatment plan was discussed by a multidisciplinary team, and aggressive palliation was deemed the most appropriate due to her significant symptoms.

At that time, her disease had progressed to compose a contiguous mass that included areas of the deep subcutaneous tissues between her visible lesions and was deemed inoperable. The effect of radiation therapy in this disease entity was not well known, but was

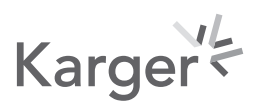




\section{Case Reports in Oncology}

\begin{tabular}{l|l}
\hline Case Rep Oncol 2021;14:17-23 \\
\hline DOI: 10.1159/000511073 & $\begin{array}{l}\text { ○ 2021 The Author(s). Published by S. Karger AG, Basel } \\
\text { www.karger.com/cro }\end{array}$ \\
\hline
\end{tabular}

Chamberlin et al:: Aneurysmal Fibrous Histiocytoma: Palliative Radiation Therapy and Targeted Therapy

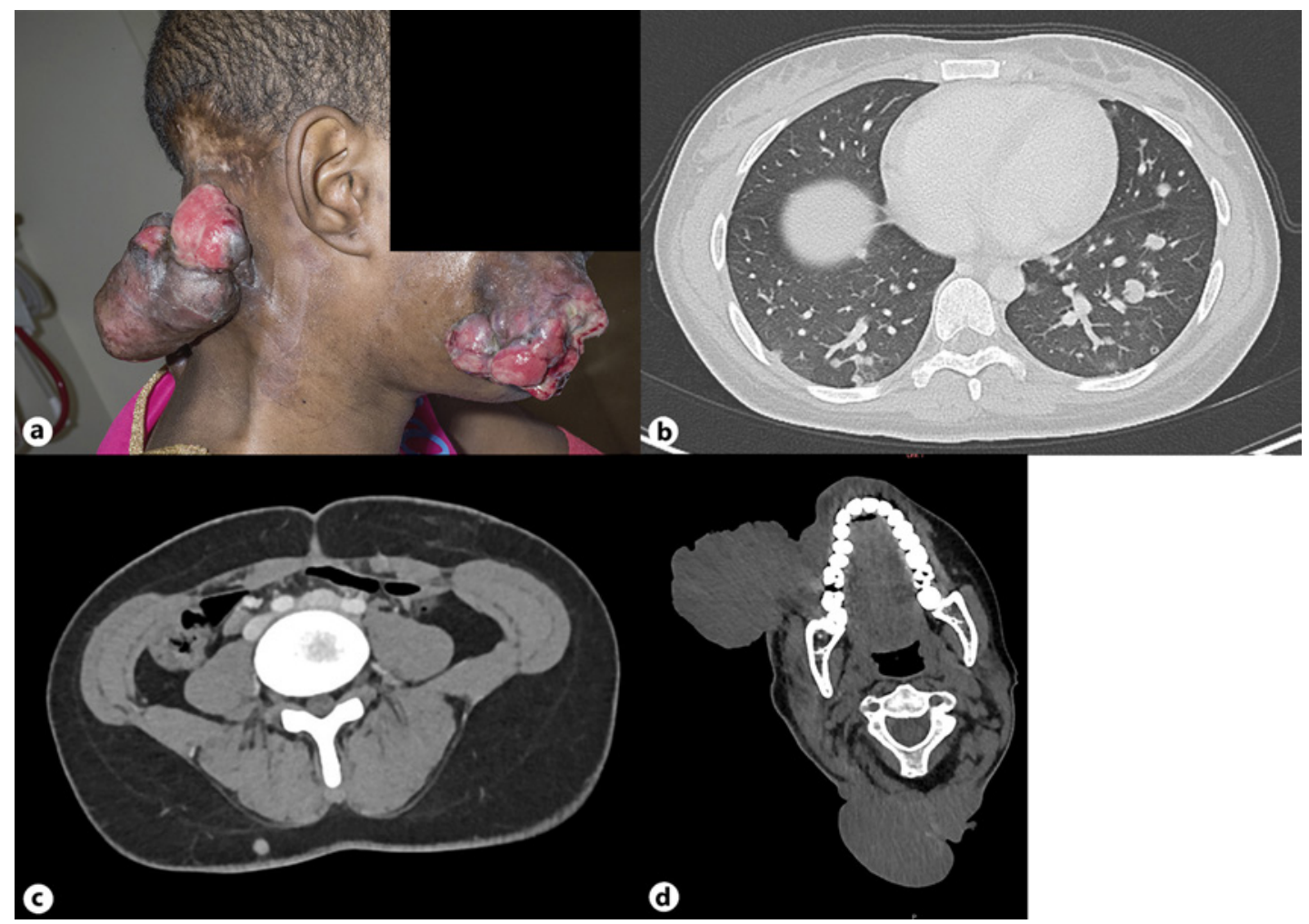

Fig. 1. a Tumor at the time of presentation. Large visible external lesions on the right cheek and occiput. b, c CT scans demonstrating pulmonary and soft tissue metastatic lesions. $\mathbf{d}$ CT scan demonstrating invasion of the tumor into the muscles of mastication.

deemed the next potential palliative option. Ultimately, given the lesion location and size as well as the clinical status of the patient, a fractionated palliative approach was undertaken. The patient's therapy began with a conformal intensity-modulated radiation therapy plan. Doses of 400 cGy per fraction were used to maximize the potential hemostatic effects of the radiation. However, after only 2 fractions, her tumor enlarged and expanded beyond the original treatment volume. She was then treated with a static field plan in 300-cGy daily fractions. Her treatment course was complicated by progressive pain and intermittent episodes of heavy venous and occasionally small arterial bleeding. She required a 2-day treatment break, and ultimately her treatment was stopped prematurely at 2,300 cGy due primarily to her progressive bleeding.

Despite the significant bleeding she experienced while receiving radiation therapy, over the following weeks, improvements in her lesions were noted (Fig. 2a-c). Her tumor decreased in size (Fig. 2d; 2 months after completing radiation), her pain had improved from baseline, and she had no episodes of clinically significant hemorrhage. Additionally, there was significant improvement in her trismus, allowing her to tolerate oral intake, leading to rapid improvement in her general health and quality of life.

This response allowed for her enrollment in a phase I trial of an oral chemotherapeutic agent; however, she eventually developed progressive disease and was taken off the study. She was then treated with the multifunctional TKI pazopanib. Pazopanib was initiated at 800 mg daily, but increased bleeding from her head and neck lesions required a dose reduction to $400 \mathrm{mg}$ daily. She developed progressive disease after 3 months. The patient was then treated with the broad-spectrum protein kinase C (PKC) inhibitor midostaurin. Midostaurin inhibits 


\section{Case Reports in Oncology}

Chamberlin et al.: Aneurysmal Fibrous Histiocytoma: Palliative Radiation Therapy and Targeted Therapy

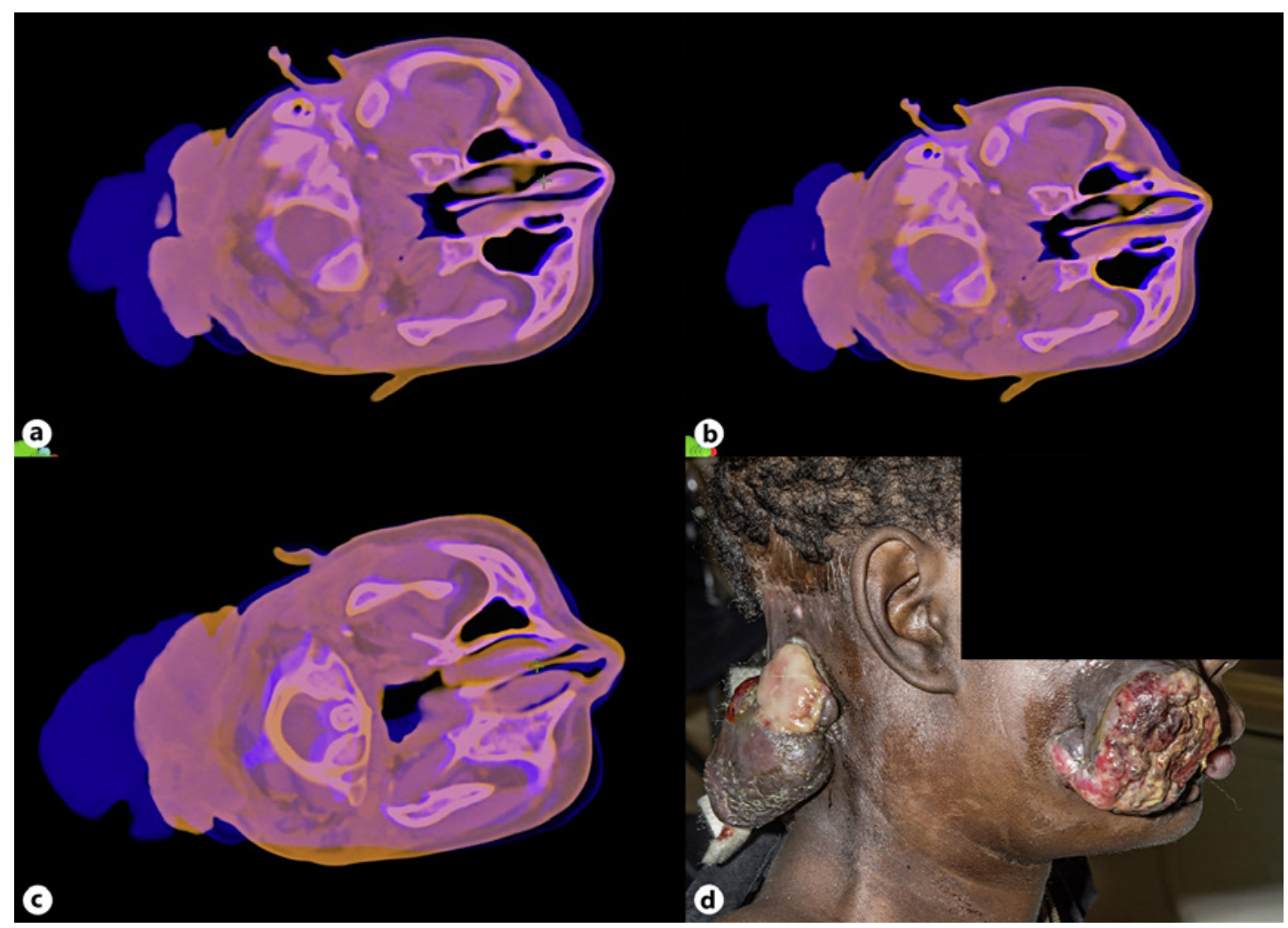

Fig. 2. a-c Color overlay of the original CT image (blue), highlighting the post-radiation response (purple) 6 weeks later at 3 axial cross-sections on CT simulation. $\mathbf{d} 2$ months after external beam radiation therapy.

a range of protein kinases including PKC isoforms and was therefore an attractive choice based on the presence of a LAMTOR-PKCD fusion. The patient stayed on midostaurin for 3 months without a decrease in tumor size.

Unfortunately, the patient ultimately did not respond to any molecular therapy regimen, and she then elected to receive comfort care measures and died of disease progression approximately 1 year later.

\section{Discussion}

$\mathrm{AFH}$ is a rare tumor that uncommonly progresses to metastatic disease. As a result, the literature available to guide potential treatment is exceedingly scarce. Surgical resection is the accepted curative approach in the localized setting; however, the increased risk of bleeding in these highly vascular tumors may limit the feasibility of this technique both with localized and with metastatic disease. Given the severity of her disease with the associated failure to thrive, she was unlikely to tolerate systemic therapy, further limiting treatment options. For this reason, palliative radiation was recommended.

Benign fibrous histiocytomas (BFHs) are a family of tumors that can be subcategorized based on morphological features - cellular, aneurysmal, epithelioid, and atypical types - and clinical manifestations - tumors of the skin, deep soft tissue, and skeleton (Fig. 3). Cytogenic analysis has been performed on uncategorized BFHs as well as on tumors diagnosed and defined as AFHs. Recurrent gene rearrangements and fusions have been identified among the 
Chamberlin et al:: Aneurysmal Fibrous Histiocytoma: Palliative Radiation Therapy and Targeted Therapy

Fig. 3. The tumor was highly cellular, composed of plump spindled cells with occasional mitoses, pools of blood, and hemosiderin-laden macrophages.

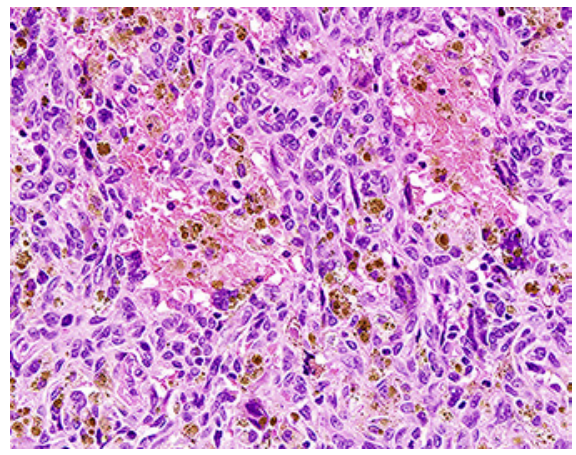

various subtypes. RNA sequencing of this tumor demonstrated a LAMTOR1-PRKCD gene fusion.

The paucity of data regarding the use of radiation therapy in this uncommon setting and makes managing this subset of patients challenging. Doyle and Fletcher [3] reported a case series of 16 patients with metastatic cutaneous fibrous histiocytoma; however, only 2 were of the aneurysmal subtype. The first patient had primary disease in the neck with initial metastatic spread to the cervical lymph node chain and soft tissue nodules in the neck (2 years from diagnosis), and subsequent spread to the lung and soft tissue of the contralateral shoulder (18 years later). He was treated with adjuvant radiation therapy to the primary site after the second local recurrence; however, the dose/fractionation data were not reported. At the most recent follow-up, the patient was alive with disease 21 years from diagnosis. The second case initially presented with disease in the shoulder, and subsequently developed axillary lymph node and pulmonary metastases at 6 and 8 years, respectively. He was treated with surgical resection of the metastatic sites and at the 15-year follow-up had no evidence of disease. Guillou et al. described a case of AFH originally presenting on the right thigh [5]. After treatment with local excision, recurrence was noted 19 years later within the ipsilateral inguinal nodes. Further imaging noted no additional disease, and the patient underwent local resection. These examples represent the typically indolent course of metastatic disease. Most cases of metastatic diseases do not present clinically until many years later and appear to be controlled with surgery, and on occasion with radiation therapy.

Information on the management and outcomes of patients with metastatic AFH is limited. However, data on the management and outcomes of patients with disease within the broad group of cutaneous fibrous histiocytoma, of which AFH is a subset, are more readily available in the literature. Therapeutic strategies including local resection, systemic chemotherapy, concurrent chemoradiotherapy, and varying courses of radiation therapy alone (stereotactic body radiation therapy and fractionated radiation therapy) have been employed [3, 5-7]. Of note, a single case report of metastatic malignant fibrous histiocytoma treated definitively with concurrent chemoradiation therapy (cisplatin, aclarubicin) resulted in a complete pathologic response at the site of treatment, though the patient ultimately died due to complications at a distant site [8]. While it is important to recognize the different pathology described in this case, it raises considerations regarding the potential role for combination therapy in this group of patients. In this setting, molecular analyses may be key in correctly identifying tumor types and tumor-specific gene targets.

In a study of 8 patients with uncategorized BFHs, frequent fusions between genes coding for membrane-association proteins (LAMTOR1, CD63, and podoplanin) with PKC isoforms $P R K C B$ and $P R K C D$ were reported [9]. PKCs are serine/threonine kinases that, upon activation, translocate and anchor to the endosomal/lysosomal/cell membranes to interact with several signaling pathways. It has been postulated that the membrane-binding domains of 
PDP, CD63, and LAMTOR1 allow for interaction of the fusion protein with the cell membrane, activating the catalytic domain of the serine/threonine kinase of PKC. Walther et al. [10] evaluated 36 cases of $\mathrm{BFH}$ for $P R K C B$ and/or PRKCD rearrangements using fluorescence in situ hybridization. Rearrangements were detected in 5 of the 36 tumors. Three cases of AFH were included in this series, and none of the 3 had a fusion. They also performed RNA sequencing on 8 additional cases of $\mathrm{BFH}$ and identified a PDPN/PRKCB gene fusion in 1 case, and a novel fusion involving a third isoform, $P R K C A$ and $K I R R E L$, which codes for a membrane-associated protein, in a second case.

Other genetic studies on AFH have revealed the presence of a $\mathrm{t}(12 ; 19)(\mathrm{p} 12 ; \mathrm{q} 13)$ translocation [11]. The breakpoint on chromosome $12 \mathrm{p}$ was determined to be between the ETV6 and PIK3C2G genes and proximal to the $B C L 3$ gene. Another study reported an AFH with a $\mathrm{t}(3 ; 11)(\mathrm{p} 21 ; \mathrm{q} 13)$ translocation that resulted in two fusion genes: LAMTOR1-PRKCD and NUMA1-SFMBT1 [12]. The former fusion was detected in our case.

\section{Conclusions}

$\mathrm{AFH}$ is a rare variant of cutaneous fibrous histiocytoma. While it can be treated definitively with local excision alone in most cases, the role of radiation therapy in the inoperable or metastatic setting is not well documented. In the case presented, durable palliative local response, symptomatic improvement, and improved quality of life were achieved after a course of moderately hypofractionated radiation therapy. Given the rarity of the nature of this disease, shared knowledge about prior treatment responses can inform future treatment approaches.

\section{Acknowledgements}

The authors would like to acknowledge all members of the radiation oncology and pediatric oncology branch who took care of this patient.

\section{Statement of Ethics}

The research was conducted ethically in accordance with the World Medical Association Declaration of Helsinki. The subject gave written informed consent to publish her case (including publication of images). The study protocol was approved by the committee on human research of the Center for Cancer Research, National Cancer Institute.

\section{Conflict of Interest Statement}

The authors have no conflicts of interest to declare.

\section{Funding Sources}

No relevant funding of any research was received for the preparation of data or the manuscript.

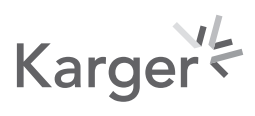




\section{Case Reports in Oncology}

\begin{tabular}{l|l}
\hline Case Rep Oncol 2021;14:17-23 \\
\hline DOI: 10.1159/000511073 & $\begin{array}{l}\text { @ 2021 The Author(s). Published by S. Karger AG, Basel } \\
\text { www.karger.com/cro }\end{array}$ \\
\hline Chamberlin et al.: Aneurysmal Fibrous Histiocytoma: Palliative Radiation Therapy and
\end{tabular}

Targeted Therapy

\section{Author Contributions}

M. Chamberlin did the main draft writing. S. Khan wrote the genetics section. C. Hernandez, J.S. Wei, and J. Khan contributed to writing parts of the genetics section and editing. J. Glod collated the edits. L. Rowe and A. Kaushal wrote the radiation sections, and A Kaushal did the overall design of the paper. M. Miettinen contributed to writing of the pathology part and the pictures. All authors read and approved the final manuscript.

\section{References}

1 Das A, Das A, Bandyopadhyay D, Mishra V, Saha A. Aneurysmal benign fibrous histiocytoma presenting as a giant acrochordon on thigh. Indian Dermatol Online J. 2015;6(6):436-8.

2 Fletcher CD. The evolving classification of soft tissue tumours: an update based on the new WHO classification. Histopathology. 2006;48(1):3-12.

3 Doyle LA, Fletcher CD. Metastasizing "benign" cutaneous fibrous histiocytoma: a clinicopathologic analysis of 16 cases. Am J Surg Pathol. 2013;37(4):484-95.

4 Gleason BC, Fletcher CD. Deep "benign" fibrous histiocytoma: clinicopathologic analysis of 69 cases of a rare tumor indicating occasional metastatic potential. Am J Surg Pathol. 2008;32(3):354-62.

5 Guillou L, Gebhard S, Salmeron M, Coindre JM. Metastasizing fibrous histiocytoma of the skin: a clinicopathologic and immunohistochemical analysis of three cases. Mod Pathol. 2000;13(6):654-60.

6 Abe T, Yamanaka K, Nakata W, Mori N, Sekii K, Yoshioka T, et al. A case of retroperitoneal malignant fibrous histiocytoma with marked response to concurrent cisplatin and radiation therapy: a case report [in Japanese]. Hinyokika Kiyo. 2007;53(4):241-6.

7 Wang Z, Wu XH, Li B, Kong QT, Shen ZT, Li J, et al. CyberKnife radiotherapy for malignant fibrous histiocytoma of the chest wall: a case report and review of the literature. Oncol Lett. 2014;7(6):1877-80.

8 Ishihama H, Ikeda Y, Shimada K. Successful chemotherapy and concurrent radiotherapy for metastatic lung. mediastinal malignant fibrous histiocytoma [in Japanese]. Nihon Kyobu Geka Gakkai Zasshi. 1995;43(10): 1757-61.

9 Płaszczyca A, Nilsson J, Magnusson L, Brosjö O, Larsson O, Vult von Steyern F, et al. Fusions involving protein kinase $\mathrm{C}$ and membrane-associated proteins in benign fibrous histiocytoma. Int J Biochem Cell Biol. 2014;53: 475-81.

10 Walther C, Hofvander J, Nilsson J, Magnusson L, Domanski HA, Gisselsson D, et al. Gene fusion detection in formalin-fixed paraffin-embedded benign fibrous histiocytomas using fluorescence in situ hybridization and RNA sequencing. Lab Invest. 2015;95(9):1071-6.

11 Botrus G, Sciot R, Debiec-Rychter M. Cutaneous aneurysmal fibrous histiocytoma with a t(12;19)(p12;q13) as the sole cytogenetic anomaly. Cancer Genet Cytogenet. 2006;164(2):155-8.

12 Panagopoulos I, Gorunova L, Bjerkehagen B, Lobmaier I, Heim S. LAMTOR1-PRKCD and NUMA1-SFMBT1 fusion genes identified by RNA sequencing in aneurysmal benign fibrous histiocytoma with $\mathrm{t}(3 ; 11)(\mathrm{p} 21 ; \mathrm{q} 13)$. Cancer Genet. 2015;208(11):545-51. 